\title{
Numerical modelling of seawater intrusion in Shenzhen (China) using a 3D density-dependent model including tidal effects
}

\author{
Wei Lu ${ }^{1}$, Qingchun Yang ${ }^{2,3, *}$, Jordi D Martín ${ }^{3}$ and Ricardo Juncosa ${ }^{3}$ \\ ${ }^{1}$ Guangzhou Institute of Geography, Guangzhou 410070, China. \\ ${ }^{2}$ Key Laboratory of Groundwater Resources and Environment, Ministry of Education, College of Environment \\ and Resources, Jilin University, Changchun 130021, China. \\ ${ }^{3}$ Escuela de Ingenieros de Caminos, Universidad de A Coruña, Campus de Elviña, 15192, A Coruña, Spain. \\ *Corresponding author.e-mail: qyang@udc.es
}

\begin{abstract}
During the 1990s, groundwater overexploitation has resulted in seawater intrusion in the coastal aquifer of the Shenzhen city, China. Although water supply facilities have been improved and alleviated seawater intrusion in recent years, groundwater overexploitation is still of great concern in some local areas. In this work we present a three-dimensional density-dependent numerical model developed with the FEFLOW code, which is aimed at simulating the extent of seawater intrusion while including tidal effects and different groundwater pumping scenarios. Model calibration, using waterheads and reported chloride concentration, has been performed based on the data from 14 boreholes, which were monitored from May 2008 to December 2009. A fairly good fitness between the observed and computed values was obtained by a manual trial-and-error method. Model prediction has been carried out forward 3 years with the calibrated model taking into account high, medium and low tide levels and different groundwater exploitation schemes. The model results show that tide-induced seawater intrusion significantly affects the groundwater levels and concentrations near the estuarine of the Dasha river, which implies that an important hydraulic connection exists between this river and groundwater, even considering that some anti-seepage measures were taken in the river bed. Two pumping scenarios were considered in the calibrated model in order to predict the future changes in the water levels and chloride concentration. The numerical results reveal a decreased tendency of seawater intrusion if groundwater exploitation does not reach an upper bound of about $1.32 \times 10^{4} \mathrm{~m}^{3} / \mathrm{d}$. The model results provide also insights for controlling seawater intrusion in such coastal aquifer systems.
\end{abstract}

\section{Introduction}

Seawater intrusion constitutes a prominent hydrological problem in many coastal areas of the world. It is generally defined as the encroachment of saline water into fresh groundwater domains in coastal aquifer settings (Werner and Simmons 2008). This phenomenon will occur when the natural equilibrium state established between fresh- and seawater is disturbed as a result of natural or artificial effects, which in turn results in the deterioration of freshwater resources affecting human, animals and agriculture and industrial resources within the contaminated zones (Xue et al. 1999).

Keywords. Seawater intrusion; coastal aquifer; numerical modelling; variable density flow; FEFLOW; China. 
Seawater intrusion is a widespread environmental issue in coastal regions throughout the world. Given that coastal zones often support some higher populated areas in the world as they generally hold the best conditions for productivity and living standards due to easy accessibility of water resources. A huge quantity of groundwater is extracted to meet the needs of population growth, economic development and other activities. Overexploitation of coastal aquifer disturbs the natural steady state established between fresh- and saltwater when the freshwater level is drawn down due to excessive pumping, the freshwater and seawater boundary moves towards inland with the subsequent salinization of areas previously located within the reduce of freshwater, seawater intrusion is characterized by its invisibility, frequent dynamic changes, harmful effects, hard or even impossible reversibility and challenging management. It threatens groundwater resources as well as the ecological environment in the coastal aquifer systems (Yuan and Liang 2001). Moreover, seawater intrusion is considered to be one of the most relevant processes degrade water quality because it raises freshwater salinity to levels exceeding the acceptable drinking standards (Hany and Akbar 2011). Therefore, it is a subject of specific concern for model researchers and managers. Due to its practical importance, the numerical modelling of seawater intrusion has attracted remarkable attention in the water resources field.

A transition zone always exists between freshand saltwater since they are miscible fluids. The transition zone will move landwards when seawater intrusion occurs. Consequently, in order to numerically simulate the seawater intrusion process, a density-dependent groundwater flow model is required if we want to track the movement and changes of the transition zone between freshwater and saltwater (Diersch and Kolditz 2002; Mao et al. 2006; Jin et al. 2009).

With the advent of powerful computers and efficient numerical codes, in the last years numerous case studies on seawater intrusion applying 3D numerical models have been presented worldwide. Werner and Gallagher (2006) used the MODHMS code to explore regional-scale process in the Pioneer Valley, Australia. Aharmouch and Larabi (2004) developed a 3D hexahedral finite element model based on the variable density flow approach and successfully applied to a field case in Morocco. Van Meir and Lebbe (2002) presented the results of a 3D density-dependent simulation for a field area along the Belgian coastal plain employing the MOCDENS3D code, which has been applied to several scenarios located in the Netherlands (Oude Essink 2001). The same code was used by Giambastiani et al. (2007), who modelled saltwater intrusion process in the unconfined coastal aquifer of Ravenna, Italy, analyzing how past and present human activities have affected the transition zone and how the forecasted sea level rise would affect the salinisation process. Jin et al. (2009) applied the SEAWAT code to investigate the extent of seawater intrusion in the Gulf Coast aquifers of Alabama, USA. Sanford and Pope (2010) performed a numerical simulation of two pumping schemes to predict the potential areas of seawater intrusion in the Eastern shore of Virginia, USA, with the U.S. Geological Survey code SEAWAT2000. A numerical simulation was carried out also by Zhang et al. (2004) to assess the impact of the choice in seaward boundary conditions on the penetration of the saltwater interface at Gooburrum, Australia, by using computer code 2DFEMFAT. Langevin et al. (2005) conducted a study to quantify flow and salinity patterns and to evaluate effects of hydrologic processes by coupling SWIFT2D and SEAWAT code and its application to the southern Everglades of Florida and northeastern Florida Bay (USA). Additionally, some other studies on seawater intrusion have been described by Andersen et al. (1988), Paniconi et al. (2001), Shoemaker and Edwards (2003), Oude Essink (2001), Langevin (2003), Qahman and Larabi (2006), Perera et al. (2008, 2009, 2010); and similar studies within the domain have been conducted by $\mathrm{Hu}$ and Jiao (2010) and Chen and Jiao (2007); however, they did not consider tidal effects and groundwater extraction scenarios.

Excessive groundwater exploitation in coastal aquifers is one of the major reasons for seawater intrusion, which contaminates freshwater resources in coastal aquifers by increasing the salinity levels of the groundwater. Seawater intrusion is identified as a critical issue that needs to be addressed in coastal hydrology, due to its irreversible effect on the water quality of coastal freshwater aquifers. The environmental problems caused by seawater intrusion have unfavourably affected economic and social development at the coastal area in Shenzhen city. Identification of the extent of seawater intrusion under different precipitation and groundwater exploitation scenarios provides useful information to prevent or mitigate seawater intrusion. We have performed a 3D model to understand the densitydependent solute transport process taking place when seawater intrusion occurs. Therefore, the purpose of this study was to quantitatively evaluate the impacts of various groundwater pumping scenarios and tidal effects on seawater intrusion.

The model developed in this study is based on the transition zone approach, which couples the groundwater flow and mass transport equation to solve the density-dependent flow. It focuses on the 
analysis of seawater intrusion in Shenzhen, one of the earliest special economic zone, and open coastal city in China. Firstly, a general description of the study area is presented. Secondly, a 3D density-dependent numerical model based on FEFLOW is described. Model calibration is based on the available records of water heads and chloride concentrations and the movement of freshwater and saltwater interface is modelled and analysed considering tidal effects and different groundwater exploitation scenarios. Finally the predicted results arising from the different scenarios considered are discussed and some conclusions are given.

\section{Description of the study area}

Shenzhen is a coastal city located in Guangdong Province, southern China. It is situated in the Pearl River Delta, north of Hong Kong, and it is bounded by Daya bay and the Zhujiang estuary to the east and west, respectively. It has a subtropical oceanic monsoon climate characterized by a distinct wet summer (May-September) with a high average rainfall (1511 mm/year), and a dry winter season (October-April) with a low average rainfall (415 mm/year). The area of interest for this study lies between the Nanshan and Futian regions connecting to Shenzhen bay at the south, covering a surface of $76 \mathrm{~km}^{2}$ (figure 1). Shenzhen Bay is located in a weak tidal area with a difference of about $3 \mathrm{~m}$ between the high and low tide levels. Shenzhen is the earliest special economic zone and open coastal city in China. Here seawater migrates into the freshwater aquifer because of groundwater over-pumping associated with the necessities arising from city development and other purposes.

\section{Hydrogeologic features}

From the hydrogeological point of view, the study area represents a fluvial-marine plain and lowheight residual hill. The main aquifers associated to the Dasha and old Yuanshatou rivers in the study area are formed with coarse sand- and gravelsize materials. In addition, a fissure system is also present and related to differential weathering effects at the surface of the bedrock. Finally, a special groundwater zone storing seawater is included in the area, which is related with manmade activities for construction works. Due to the high degree of variability and lack of continuities, the hydrogeological formation of the study area is based on both lithology and hydrogeologic properties. Four vertical layers or units are therefore identified. Their properties are given next aquifer system is determined, which are described as follows:

(1) Aquitard I: It is mainly formed with manmade earthfills, muddy clay and silt, its thickness ranges from approximately 2 to $7 \mathrm{~m}$, with the thickness reduces south towards north.

(2) Quaternary porous aquifer: It consists of predominantly medium-coarse grained sands and

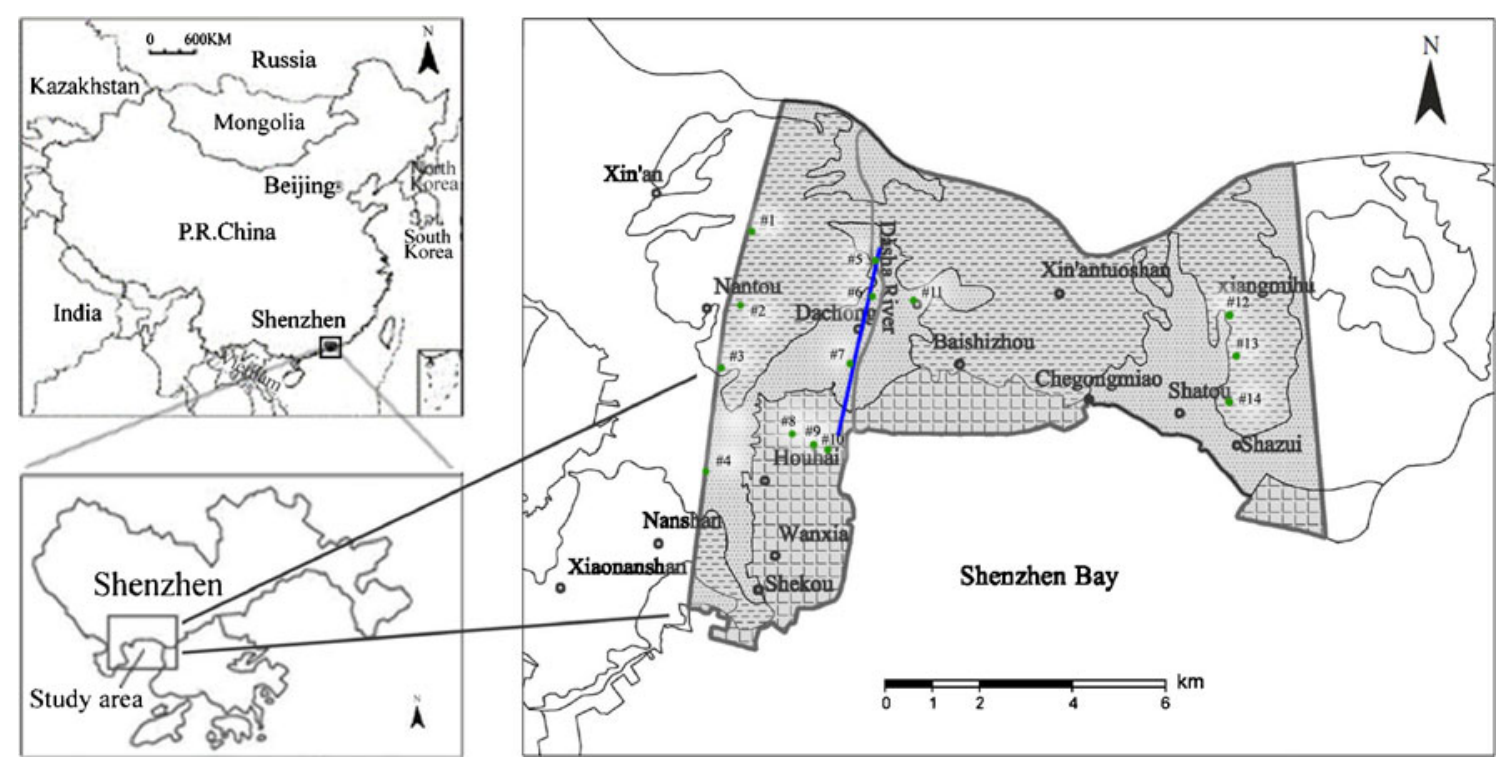

Figure 1. Locality map and domain outline of the study area (the blue line indicates the location of cross-section, the green circle with \#n represents the location of the observation well). 


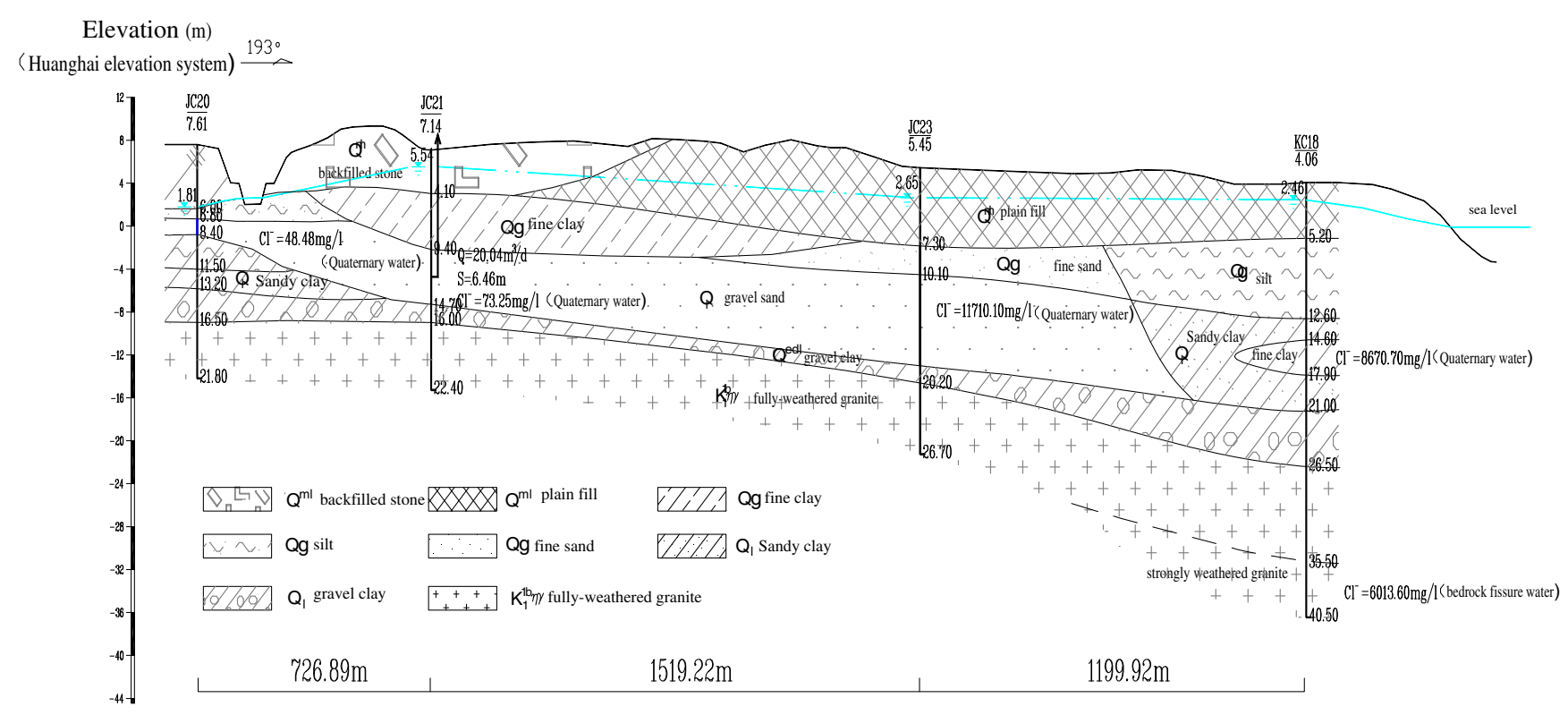

Figure 2. Geological profile of the study area.

gravels, with an average thickness between 1.5 and $15 \mathrm{~m}$. It is partly confined due to the overlying of the aquitard.

(3) Aquitard II: It is composed by gravel, silty clay and silt and it has low hydraulic conductivity. The thickness ranges from 5 to $20 \mathrm{~m}$.

(4) Fissure aquifer: Its dominant lithology is granite, mixed rock and quartz sand. The material represents a highly weathered fissured rock. Fissures are highly conductive. And it is a major water-bearing system. The thickness of this unit ranges from $6.5 \mathrm{~m}$ in the south to about $43 \mathrm{~m}$ in the north. The geological profile of the study area is illustrated in figure 2 .

\section{Regional hydrologic characters}

\subsection{Groundwater recharge}

The recharge sources are predominantly rainfall, lateral seepage from the fissure system of the granite bedrock at the north and river seepage. Rainfall recharge is the primary influx due to the fairly weak surface runoff resulting from the flat topography. However, great differences in the intensity of recharge exist because of the spatial variability of the aquifers. Fissure water in the study area is mainly recharged through infiltration of precipitation and lateral seepage from the granite outcropping area in the northern part of the studied domain. In the region of reclamation, water is stored in the upper earthfills, which receive rainfall recharge and lateral seepage.
The lower part is maintained almost at a stable state.

\subsection{Groundwater runoff and discharge}

The general trend of groundwater runoff in the study area is controlled by the topography, which is characterized by a flat runoff southward to Shenzhen bay. Three types of discharge are found in the study area: (a) groundwater discharge, (b) evaporation (phreatic evaporation and plant transpiration) and (c) regional artificial discharge (groundwater extraction).

\section{Numerical model}

\subsection{Simulation code}

Groundwater flow system is unsteady since the movement of groundwater is subject to Darcy's law under the conditions of normal temperature and pressures, whose inputs and outputs are temporally and spatially variable parameters. Due to the density differences between seawater and freshwater, the hydraulic dispersion and molecular diffusion are dominant phenomena causing seawater intrusion.

The first attempts to model the densitydependent miscible seawater/freshwater were carried out by Henry (1964) and Pinder and Cooper (1970). This approach is preferable to the common assumption that the two-phase fluid flow is separated by a sharp interface associated to 
the Ghyben-Herzberg relationship (Werner and Gallagher 2006). For our work, a 3D densitydriven numerical model was adopted considering the groundwater flow movement and flow exchanges among shallow and deep aquifers which is represented by inter-layered cross flow (Sun 1981).

The code employed in the simulation was FEFLOW (finite element subsurface FLOW system). This is a modular 3D finite element groundwater flow model, capable of simulating processes that are transient or steady-state, fluid densitycoupled or linear, and flow and mass, flow and heat or completely coupled thermohaline transport processes in subsurface water resources (Diersch 2002).

The governing equations for a mixed variable density flow, in terms of freshwater head and salt movement of a miscible freshwater-seawater, which are shown in table 1 (Huyakorn et al. 1987). And the relevant parameters are defined in table 2 .

In addition to the flow equation, the second partial differential equation is required to describe solute transport in the system due to the hydrodynamic dispersion (Bear 1979), which is expressed by equations listed in table 3 , and table 4 presents the definitions of symbols used in previous table except the same denotations of some parameters as in table 1.

The movement of groundwater flow will cause the redistribution of solute concentrations in the system, which in turn changes the density and ultimately, influences groundwater flow patterns. Therefore, groundwater flow and solute transport are coupled processes, and these two should be solved jointly. Galerkin finite element method is adopted to solve these two equations, considering the possible effect of numerical dispersion and oscillation of temporal and spatial discretization, Shock Capturing (SC) and Forward Euler (FE), automatic time step control via predictor-corrector schemes in the FEFLOW subroutines are selected to perform simulations (table 5).

Seepage velocity can be written as:

$$
v_{j}=k_{i j}\left(\frac{\partial H}{\partial x_{j}}+\eta C e_{j}\right) .
$$

According to Darcy's law, the transfer rate and coefficient between river and groundwater can be obtained through equations (2) and (3), and the solute flux can be calculated by equation (4)

$$
\begin{gathered}
q_{n a}=-K_{0}^{\text {in }} \frac{h_{2}^{R}-h}{d} \\
\Phi_{h}^{\text {in }}=\frac{K_{0}^{\text {in }}}{d} \\
q_{n c}=-f_{C}\left(C_{3}^{R}-C\right)
\end{gathered}
$$

Table 1. Flow equation for variable density flow.

\begin{tabular}{ll}
\hline Variable density flow equation & $\frac{\partial}{\partial x_{i}}\left[K_{i j}\left(\frac{\partial H}{\partial x_{j}}+\eta C e_{j}\right)\right]=S_{s} \frac{\partial H}{\partial t}+\phi \eta \frac{\partial C}{\partial t}-\frac{\rho}{\rho}$ \\
Initial conditions & $H\left(x_{i}, 0\right)=H_{0}\left(x_{i}\right)$ \\
Dirichlet boundary & $\left.H\left(x_{i}, t\right)\right|_{\Gamma_{1}}=H_{B}\left(x_{i}, t\right)$ \\
No-flux boundary & $-\left.v_{i} n_{i}\right|_{\Gamma_{2}}=0$ \\
Neumann boundary condition & $-\left.v_{i} n_{i}\right|_{\Gamma_{2-1}}=\left(\frac{\rho_{0}}{\rho} W^{\prime}-\frac{\rho^{*}}{\rho} \mu_{d} \frac{\partial H^{*}}{\partial t}\right) n_{3}$ \\
& $-\left.v_{i} n_{i}\right|_{\Gamma_{2-2}}=\frac{\rho_{B 2}}{\rho} v_{B 2}$ \\
\hline
\end{tabular}

Table 2. Symbols used in table 1.

$K_{i j} \rightarrow$ principal components of hydraulic conductivity along the x, y, and $\mathrm{z}$ axes, respectively

$H \rightarrow$ equivalent freshwater hydraulic head; $\quad x_{i}, x_{j}(i, j=1,2,3) \rightarrow$ Cartesian coordinates

$\eta \rightarrow$ density coupling coefficient

$S_{s} \rightarrow$ specific storage of the porous medium

$v_{i} \rightarrow$ actual seepage velocity;

$t \rightarrow$ time

$q \rightarrow$ a volumetric flow rate per unit volume of aquifer representing sources and/or sinks of water

$\phi \rightarrow$ porosity;

$\rho, \rho_{0} \rightarrow$ density of salt-fresh water and fresh water

$H_{0} \rightarrow$ initial water head;

$H_{B} \rightarrow$ specified water head at Boundary $\Gamma_{1}$

$H^{*} \rightarrow$ reference water table;

$\nu_{B 2} \rightarrow$ in and/or out flux at specified boundary per unit of area

$\rho^{*} \rightarrow$ water density at the transition zone;

$\rho_{B 2} \rightarrow$ flow density at specified-flux boundary

$\mu_{d} \rightarrow$ gravitational specific yield;

$n_{i} \rightarrow$ vertical vector at $x_{i}$ direction for boundary $\Gamma_{2}, \Gamma_{2-1}, \Gamma_{2-2}$

$\Gamma_{1}, \Gamma_{2}, \Gamma_{2-1}, \Gamma_{2-2} \rightarrow$ boundary of specified flux, no-flux, phreatic water and dispersion flux 
Table 3. Solute transport equation and the responding initial and boundary conditions.

\begin{tabular}{ll}
\hline Transport equation & $\frac{\partial}{\partial x_{i}}\left(D_{i j} \frac{\partial C}{\partial x_{j}}\right)-\frac{\partial\left(u_{i} C\right)}{\partial x_{i}}=\frac{\partial C}{\partial t}-\frac{q}{\phi} C^{*}$ \\
Initial conditions & $C\left(x_{i}, 0\right)=C_{0}\left(x_{i}, t\right)$ \\
Dirichlet bondary & $\left.C\left(x_{i}, t\right)\right|_{\Gamma_{1}}=C_{B}\left(x_{i}, t\right)$ \\
No-flux boundary & $\left.\left(D_{i j} \frac{\partial \mathrm{C}}{\partial x_{j}}+u_{i} C\right) \phi n_{\mathrm{i}}\right|_{\Gamma_{2}}=0$ \\
Neumann boundary condition & $-\left.D_{i j} \frac{\partial C}{\partial x_{j}} n_{i}\right|_{\Gamma_{2-1}}=\left(1-\frac{\rho^{*}}{\rho}\right) \frac{C}{\phi} \mu_{d} \frac{\partial H^{*}}{\partial t} n_{3}+\frac{W^{\prime}}{\phi}\left(\frac{\rho_{0}}{\rho} C-C^{\prime}\right) n_{3}$ \\
& $-\left.D_{i j} \frac{\partial C}{\partial x_{j}} n_{i}\right|_{\Gamma_{2-2}}=\frac{v_{B}}{\phi}\left(\frac{\rho_{B}}{\rho} C-C^{\prime \prime}\right) n_{3}$ \\
\hline
\end{tabular}

Table 4. Symbols used in table 3.

\begin{tabular}{ll}
\hline$D_{i j}$ & apparent hydrodynamic dispersion tensor $(i, j=1,2,3)$ \\
$H$ & equivalent freshwater hydraulic head \\
$C, C_{0}, C_{B}$ & solute concentration in the active phase, initial concentration and the concentration \\
& at boundary $\Gamma_{1}$ \\
$u_{i}$ & average seepage velocity \\
$\rho, \rho_{0}$ & density of salt-fresh water and fresh water, which is assumed to be a linear function \\
& of the concentrations in the model \\
$\nu_{B 2}$ & in and/or out flux at specified boundary per unit of area \\
$\rho_{B}$ & flow density at specified-flux boundary \\
$H^{*}$ & reference water table \\
$\rho^{*}$ & water density at the transition zone \\
$C^{*}$ & concentration of water from source or/and sink \\
$\mu_{d}$ & gravitational specific yield \\
$n_{i}$ & vertical vector at $x_{i}$ direction for boundary $\Gamma_{2}, \Gamma_{2-1}, \Gamma_{2-2}$ \\
$\Gamma_{1}, \Gamma_{2}, \Gamma_{2-1}, \Gamma_{2-2}$ & boundary of specified concentration, no-flux, phreatic water and dispersion flux \\
$C^{\prime \prime}$ & concentration of in and/or out water at specified-flux boundary \\
\hline
\end{tabular}

Table 5. Symbols used in equations (1), (2) and (3).

\begin{tabular}{ll}
\hline$q_{n a}-$ transfer rate of river and groundwater & $q_{n c}-$ solute flux \\
$\Phi-$ transfer coefficient & $f_{C}-$ transfer rate of injection or extraction \\
$K_{0}^{\text {in }}, K_{0}^{\text {out }}-$ hydraulic conductivity of river bed & $C_{3}^{R}-$ solute concentration of river \\
$\quad$ for river-groundwater recharge or discharge & \\
$h_{2}^{R}-$ river table & $C$ - solute concentration beneath the riverbed \\
$h$ - groundwater table & $d$-thickness of river bed \\
\hline
\end{tabular}

\subsection{Boundary and initial conditions}

Since the model is set up to simulate seawater intrusion involving both groundwater flow and solute transport process in a coastal aquifer, boundaries for these two processes are required. For the flow model, the boundary conditions include specified flux to simulate recharge and evapotranspiration at the surface and no-flux at the bottom due to the contact with granite. Dasha river intersects the porous aquifer, where a headdependent flux is specified to simulate the leakage between the two water bodies. A fairly thick overlying alluvial layer overlies the granite in the bottom part of the study area. The movement of groundwater is perpendicular to the east and west sides, where a no-flow boundary is assigned. Seawater is contact with the aquifer at the south. To 
the authors' knowledge, there is no extraction cone in the study area and the tidal effect is the main factor inducing seawater intrusion. Thus, a Dirichlet prescribed-head boundary condition was prescribed, including a time series function depending on the periodicity and magnitude of tides.

For the solute transport model, chloride concentration was selected due to its conservative nature and tracer-bearing capacity. The boundary condition are Dirichlet constant concentration, where freshwater is in contact with seawater on the south, the chloride concentration is set to 30 and
$13000 \mathrm{mg} / \mathrm{l}$ in the freshwater and seawater, respectively. On the north side, a Neumann prescribedflux boundary is imposed. This is based on the initial and final fluxes observed in the simulation period. For the remaining two boundaries, no-flux boundaries were assigned.

The initial conditions for the model adopt the observed water level and concentration data during May 2008, which was processed through interpolation and extrapolation methods. Then, initial flow and concentration field are obtained by simulating the virtual water levels assigned to the model.

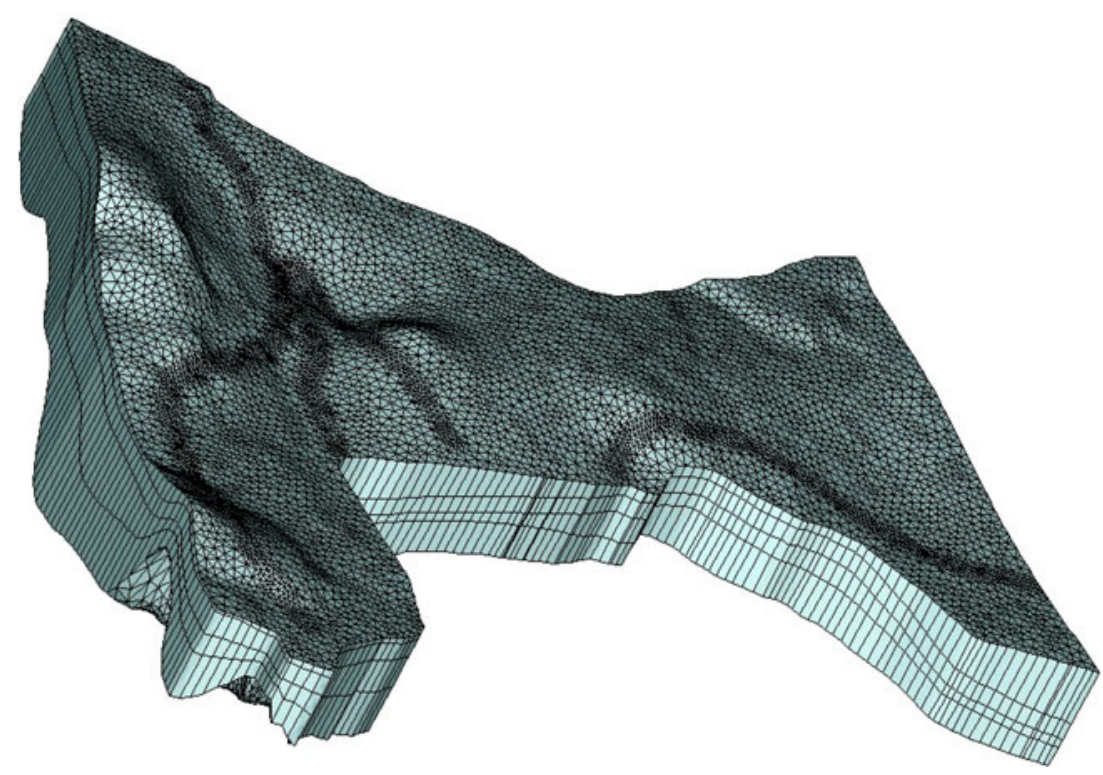

Figure 3. Spatial discretisation for the three-dimensional numerical model.
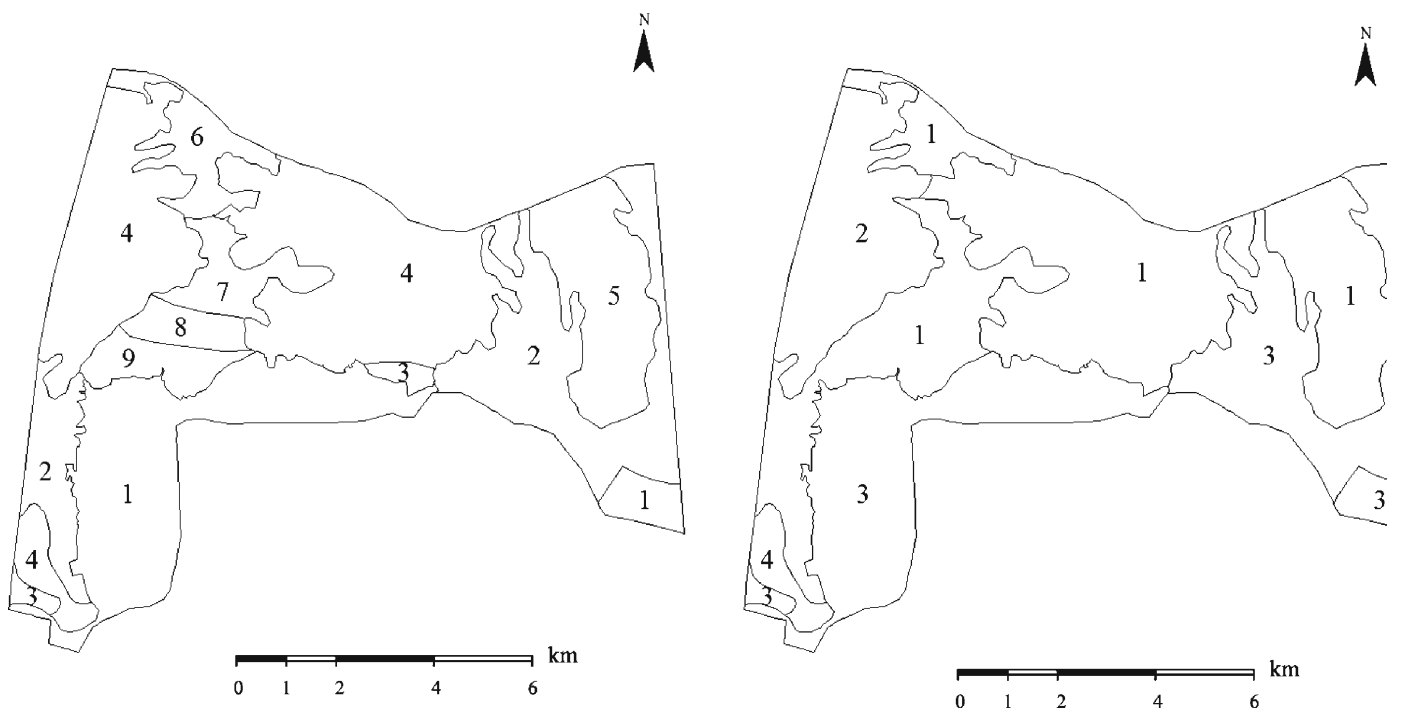

Figure 4. Zonations of hydrogeologic parameters (left) and precipitation recharge coefficient (right). 
Table 6. Hydrogeologic parameters associated to different zones of the model domain.

\begin{tabular}{|c|c|c|c|c|c|c|c|c|}
\hline Layer & Zone & $\begin{array}{c}K_{x x}, K_{y y} \\
\left(\times 10^{-4} \mathrm{~m} / \mathrm{s}\right)\end{array}$ & $\begin{array}{c}K_{z z} \\
\left(\times 10^{-4} \mathrm{~m} / \mathrm{s}\right)\end{array}$ & $S_{y}$ & $S_{s}(1 / \mathrm{m})$ & $a_{L}(\mathrm{~m})$ & $a_{T}(\mathrm{~m})$ & $\Phi$ \\
\hline \multirow[t]{4}{*}{1} & 1 & 0.004 & & 0.10 & 0.0001 & 0.10 & & 0.05 \\
\hline & $2-3$ & 0.030 & & 0.12 & 0.0001 & 0.10 & & 0.05 \\
\hline & $4-5$ & 0.019 & & 0.11 & 0.0001 & 0.08 & & 0.05 \\
\hline & $6-9$ & 0.040 & & 0.12 & 0.0001 & 0.15 & & 0.05 \\
\hline \multirow[t]{9}{*}{2} & 1 & 0.960 & \multirow[t]{9}{*}{$0.1 K_{x x}$} & 0.28 & 0.0024 & 9.00 & \multirow[t]{9}{*}{$0.1 a_{L}$} & 0.21 \\
\hline & 2 & 1.920 & & 0.30 & 0.0036 & 6.50 & & 0.08 \\
\hline & 3 & 1.800 & & 0.25 & 0.0030 & 3.50 & & 0.10 \\
\hline & 4 & 0.800 & & 0.08 & 0.0030 & 0.50 & & 0.08 \\
\hline & 5 & 0.800 & & 0.08 & 0.0030 & 0.25 & & 0.08 \\
\hline & 6 & 0.970 & & 0.12 & 0.0036 & 6.00 & & 0.08 \\
\hline & 7 & 1.500 & & 0.15 & 0.0031 & 8.80 & & 0.25 \\
\hline & 8 & 2.880 & & 0.25 & 0.0033 & 8.60 & & 0.23 \\
\hline & 9 & 4.200 & & 0.33 & 0.0020 & 10.10 & & 0.30 \\
\hline \multirow[t]{2}{*}{3} & $1-2$ & 0.001 & & 0.05 & 0.0001 & 0.48 & & 0.10 \\
\hline & $3-9$ & 0.0005 & & 0.13 & 0.0001 & 0.24 & & 0.08 \\
\hline \multirow[t]{6}{*}{4} & 1 & 0.500 & & 0.11 & 0.0031 & 2.00 & & 0.10 \\
\hline & 2 & 0.700 & & 0.15 & 0.0046 & 2.50 & & 0.10 \\
\hline & 3 & 0.006 & & 0.14 & 0.0039 & 2.50 & & 0.10 \\
\hline & 4 & 0.800 & & 0.14 & 0.0039 & 2.50 & & 0.10 \\
\hline & 5 & 0.530 & & 0.14 & 0.0039 & 1.25 & & 0.10 \\
\hline & $6-9$ & 1.100 & & 0.15 & 0.0046 & 1.60 & & 0.10 \\
\hline
\end{tabular}

\subsection{Spatial and temporal discretization}

The model domain is spatially discretized into triangle grid with 32,990 nodes and 51,140 elements, as illustrated in figure 3 . The grid sizes for the seawater intrusion line and river section are refined, and the observation boreholes are set on the nodes. Forward/backward Euler automatic time step is used for the temporal discretization in the model.

\subsection{Model calibration}

Model calibration has a start time at May 2008, while the end time was set to December 2009. The data used in the calibration included the corresponding observed head and chloride concentration from 14 observation boreholes. A trialand-error method is employed to calibrate the numerical model, adjusting first the zonation, hydraulic conductivities, specific storage and storativity until the calculated water tables match well the observed values. The vertical hydraulic conductivity is estimated as $10 \%$ of the horizontal hydraulic conductivity. Following transport parameters, diffusion coefficients were estimated with the water flow parameters as fixed values. The estimated longitudinal dispersivity was set as $10 \%$ of the transverse one. The calibrated zonations and parameters are illustrated in figure 4 , tables 6 and 7, respectively. A qualitative and a quantitative measure are applied to validate the model reliability. The qualitative measure compares the modelled isolines (figure 5), with the interpolated versus observations using a regression analysis and the quantitative one in a regression analysis of the calculated and scattered observed ones (figure 6). We can observe in both figures that the model reasonably reproduces the observed values, and that the correlation coefficient of computed and observed values is close to one and well within both sides of the $95 \%$ confidential lines. One observation well, \#4, was selected as a representative one to show the goodness-of-fit (figure 7), and can observe that the computed values match the measured ones very well for this well.

Table 7. Precipitation recharge coefficient (PRC) corresponding to each zone of the model domain.

\begin{tabular}{ccccc}
\hline Zone & 1 & 2 & 3 & 4 \\
\hline PRC & 0.20 & 0.12 & 0.08 & 0.03 \\
\hline
\end{tabular}



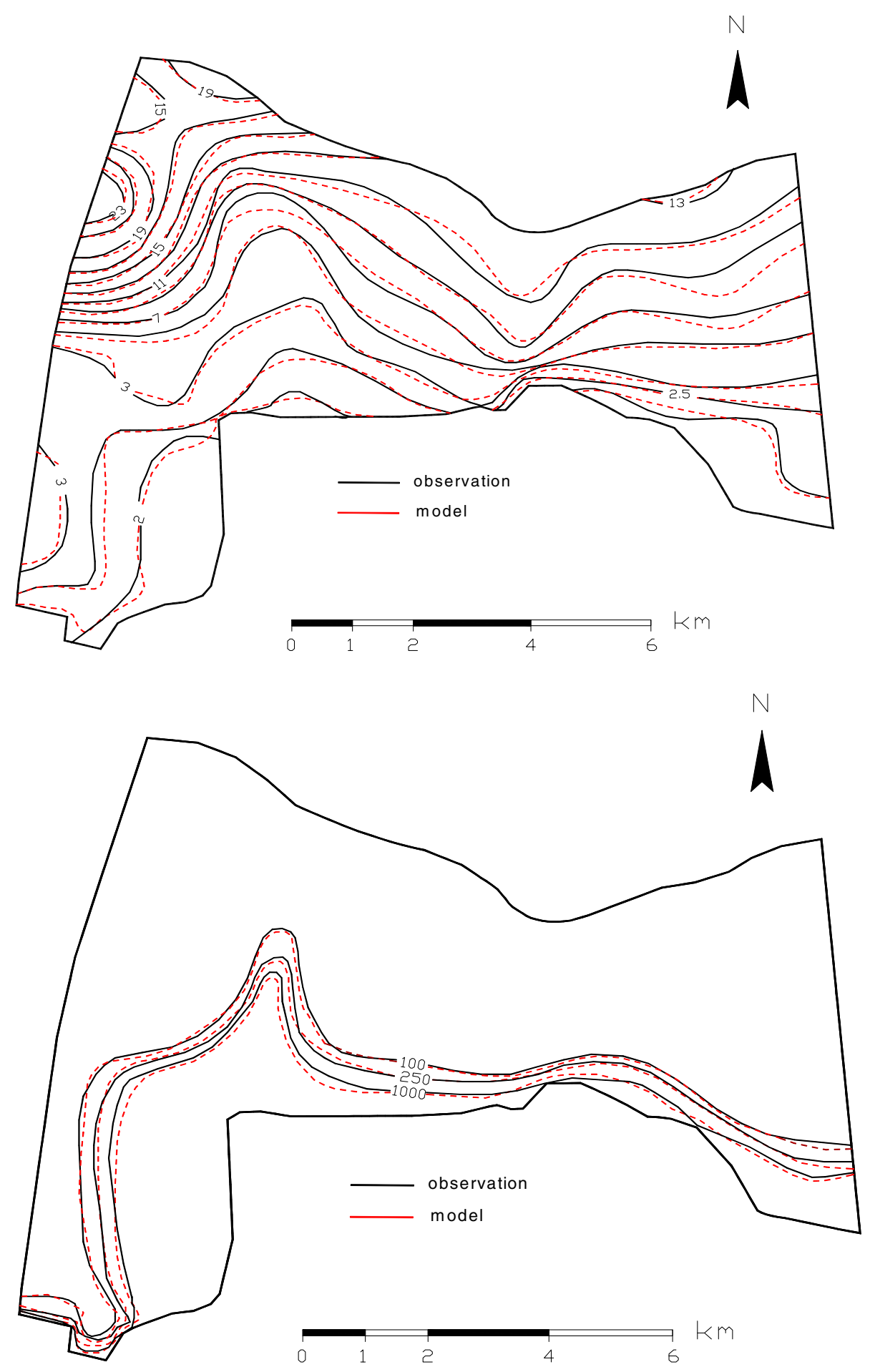

Figure 5. Comparison of observed (solid line) vs. calculated (dashed line) head (top) and concentration (bottom) contours for porous aquifer.

\section{Prediction of seawater intrusion}

In this section, tidal fluctuations and different groundwater exploitation schemes are analysed in order to forecast the extent of seawater intrusion in the study area.

\subsection{Extent of seawater intrusion resulting from the tidal effect}

Water heads in the porous aquifer are modelled under the conditions of high, medium and low tide levels, which is illustrated in figure 8. One 

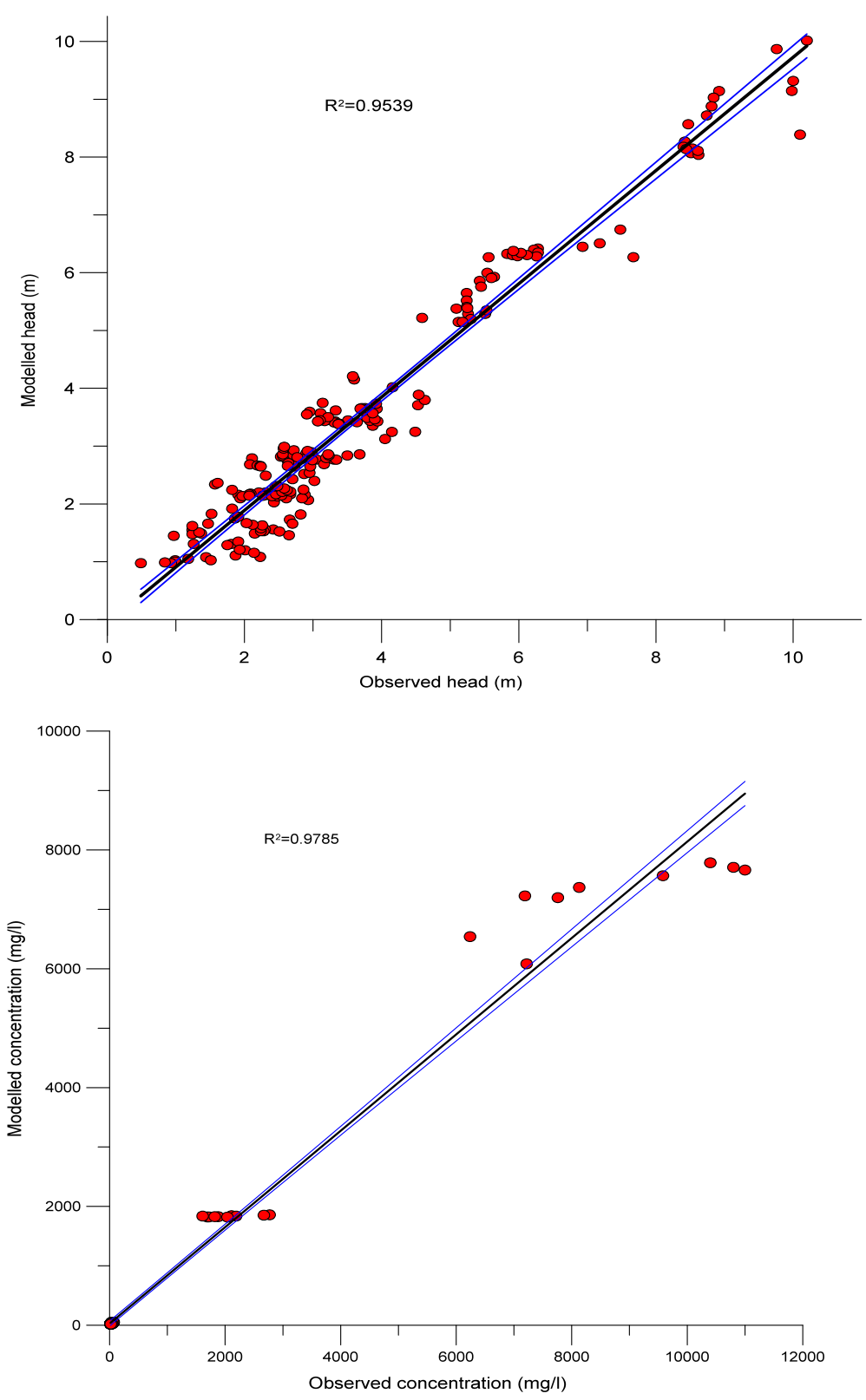

Figure 6. Comparison of observed vs. calculated water heads (top) and chloride concentrations (bottom).

can see that groundwater heads show a responsive feedback effect on the variations of seawater level caused by the tidal fluctuations, particularly near the Dasha river estuarine. In order to analyse the tidal effects on seawater intrusion, a profile along Dasha river was selected (the blue line indicated in figure 1) to demonstrate how chloride concentration evolves. Parts (d), (e) and (f) in figure 9 show that the groundwater level gradually reduced towards the entrance of Shenzhen Bay. Correspondingly, parts (a), (b) and (c) illustrate that chloride concentration enriched mainly at the entrance and gradually reduced towards north. And the highest concentration appears at the Dasha river estuarine. The largest intrusion plume peak responds to the high tide level; however, an interesting phenomenon is that chloride concentration is higher at a distance of $1.5 \mathrm{~km}$ to sea entrance; the explanation maybe the topographical effect, since the elevation of this point is relatively low, so chloride is prone to be retained thereby.

\subsection{Seawater intrusion with different groundwater exploitation scenarios}

Prediction of the extent of seawater intrusion is performed by running the calibrated 3-year model forward using the parameters given in tables 1 and 2. Based on current pumping rate, 


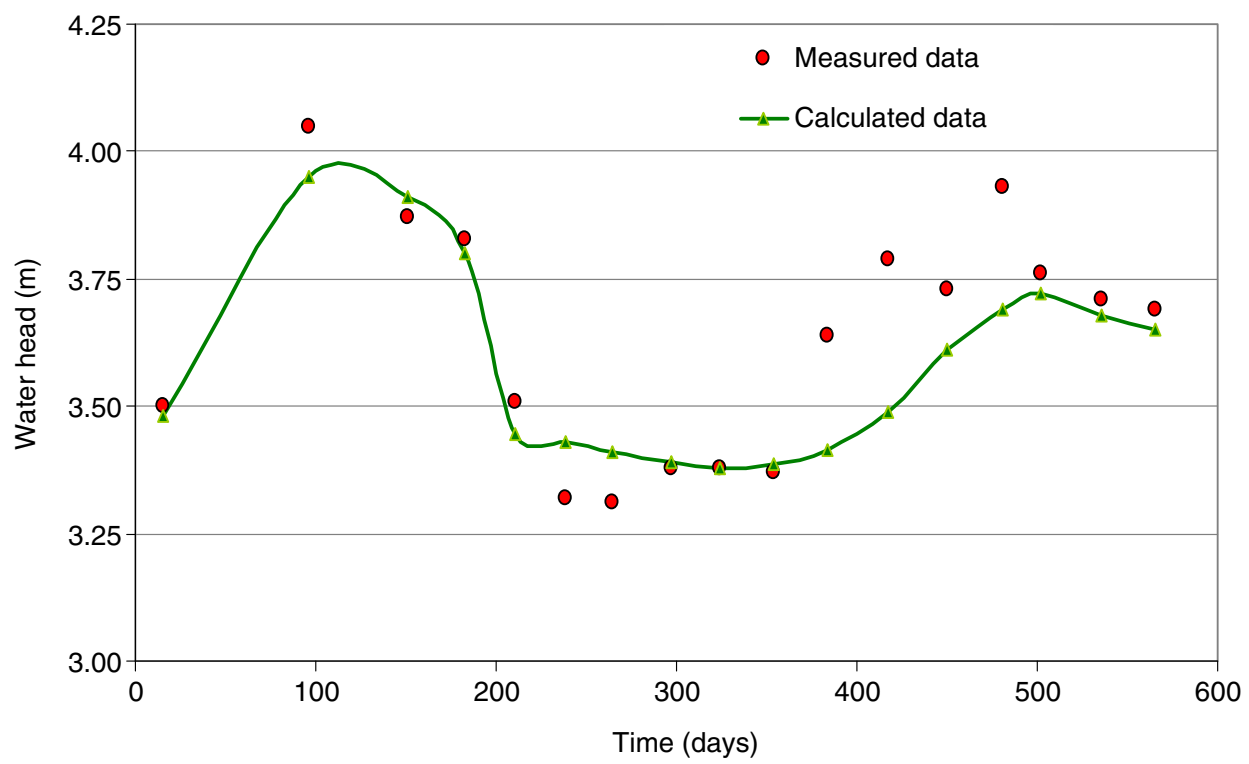

Figure 7. Comparison of measured data and calculated data for well \#4.

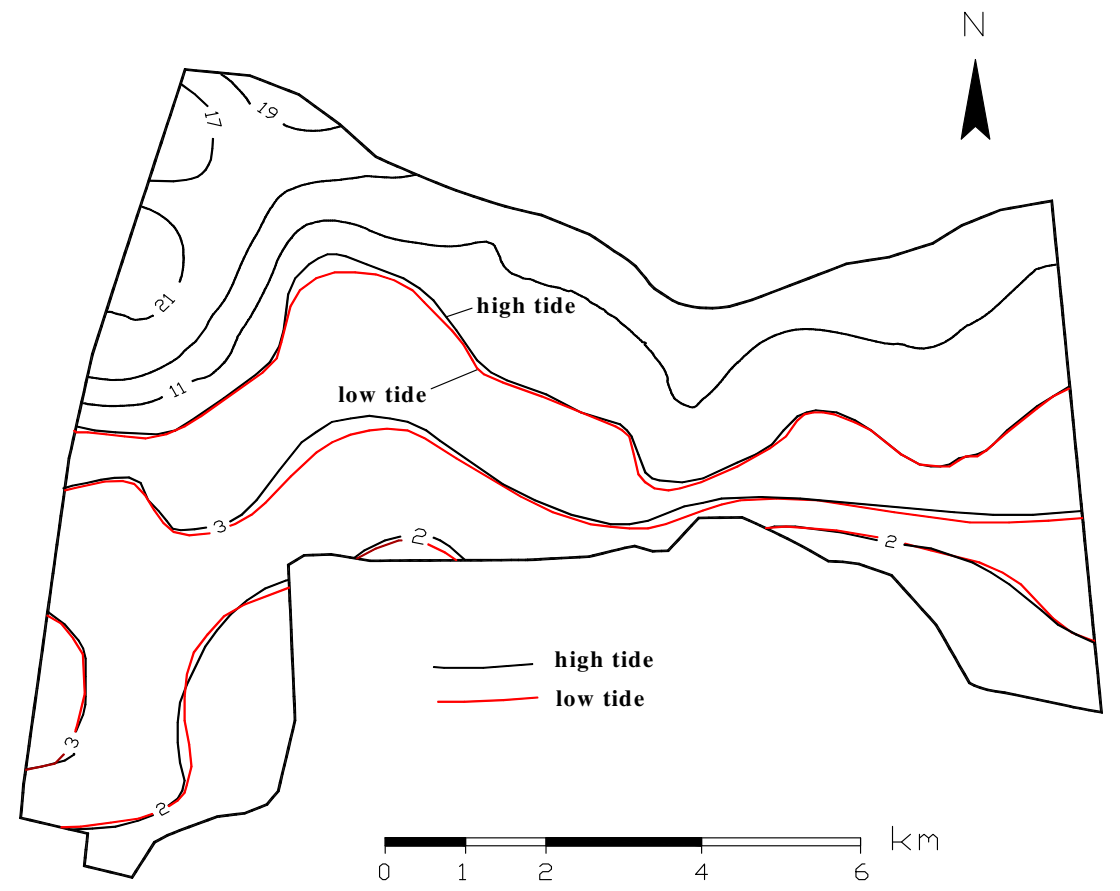

Figure 8. Changes of groundwater levels with different tide levels.

$5.5 \times 10^{3} \mathrm{~m}^{3} / \mathrm{d}$, of which $2.5 \times 10^{3} \mathrm{~m}^{3} / \mathrm{d}$ comes from basement fissure aquifer, different groundwater pumping scenarios have been developed to predict water level and chloride concentration at the end of 2013: $3.5 \times 10^{3}$ and $8.5 \times 10^{3} \mathrm{~m}^{3} / \mathrm{d}$ are designed. The results are shown in figure 10 .

We can observe that the seawater migrates inland almost similarly for current and decreased groundwater extraction. However, when pumping rate is increased, the extent of seawater intrusion becomes greater at the west boundary, while in the east seawater shows a recession tendency. This is caused by exploitation in an already saline area and water level in pumping well declines, which in turn results in increase of hydraulic gradient from upstream of pumping well towards the sea and the subsequent seawater recession. This result indicates that extraction of saline water might be an efficient controlling measure to mitigate seawater intrusion. 

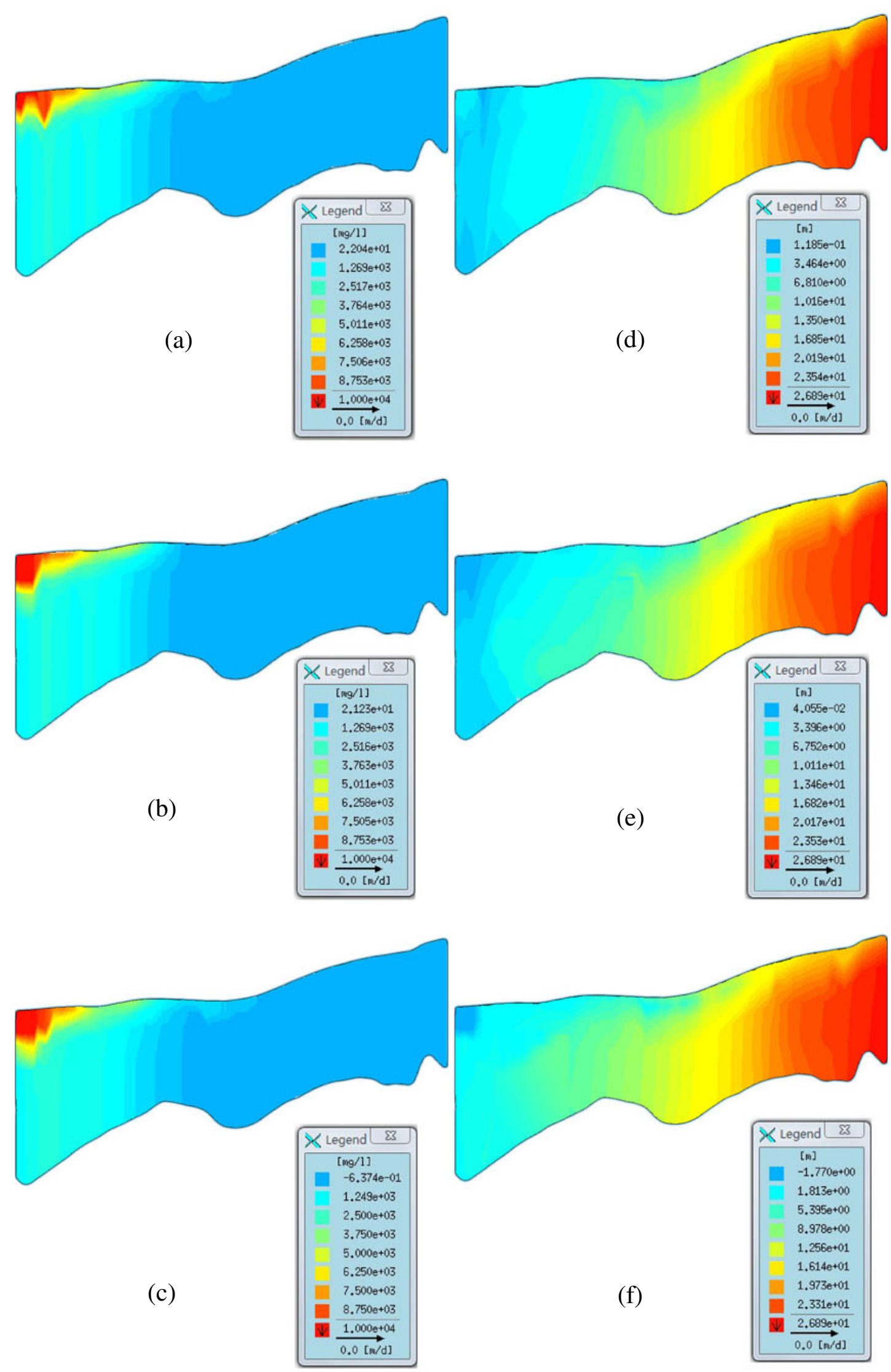

Figure 9. Predicted extension of seawater intrusion for (a) high, (b) medium and (c) low tide level. 

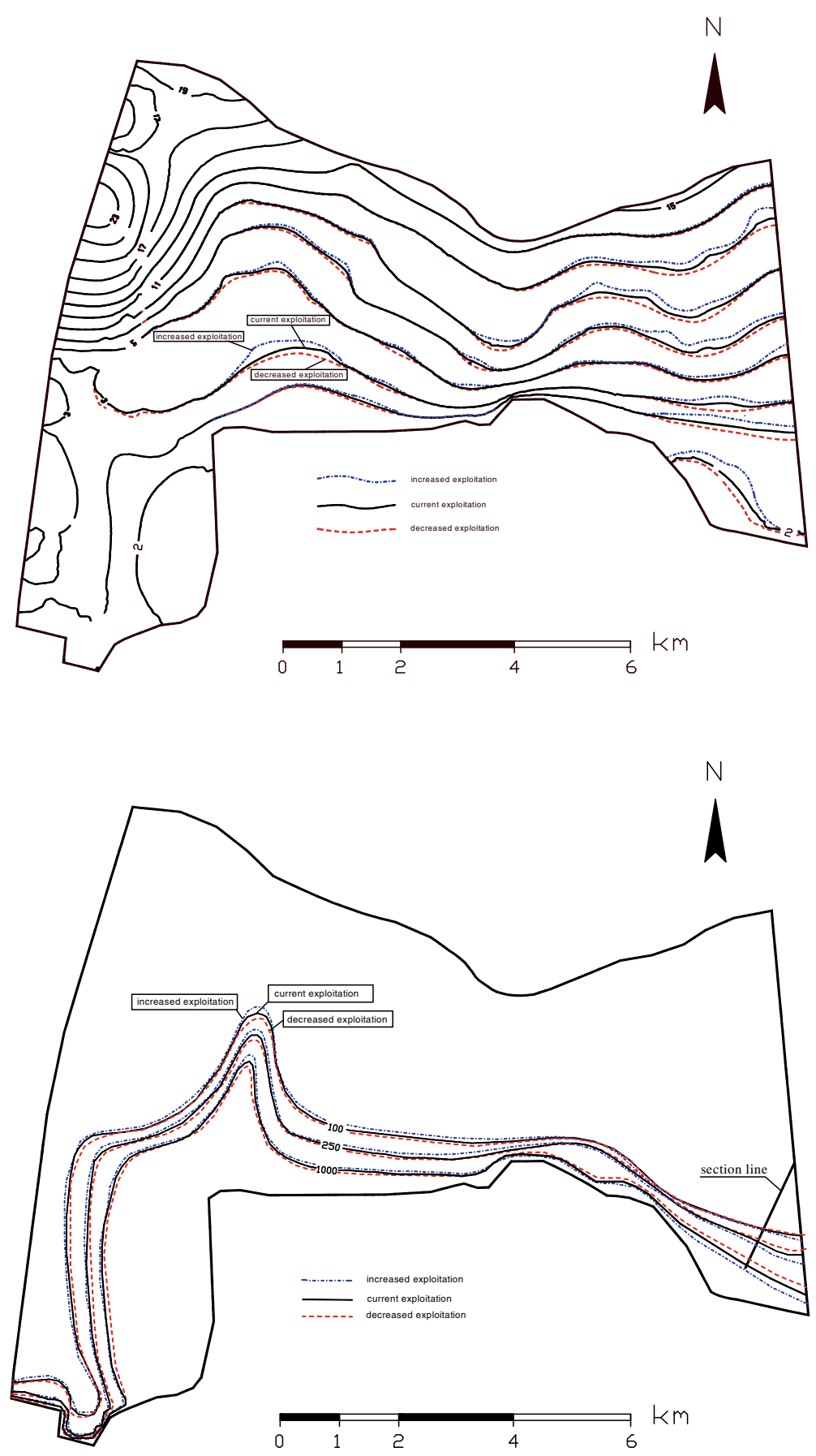

Figure 10. Comparisons between simulated groundwater levels (upper) and concentrations (lower) under different pumping schemes at the end of 2013.

To estimate the exploitation potential in the study area, a different exploitation was tried in the simulation and see the extent of seawater intrusion: for exploitation within a certain range, there is not significant seawater intrusion. $1.32 \times 10^{4} \mathrm{~m}^{3} / \mathrm{d}$ is the value where seawater intrusion occurs suddenly with great extent, so it is considered as the maximum exploitation. From the lower part of figure 9, we could conclude that the extent of seawater intrusion will be reduced as long as groundwater exploitation is not expanded.

\section{Conclusions}

A 3D density-dependent numerical model has been developed to predict the future extent of seawater intrusion. To do that, we have to analyse and conceptualize the hydrogeological setting of the study area. The conceptualizations assume non-steady state, non-homogeneous and heterogeneous aquifer properties. Model calibration was carried out by using groundwater table and chloride concentrations from a total of 14 
observation wells. A fairly good match between the observed and computed values was achieved. Model prediction was then performed based on the calibrated parameters. The predicted extent of seawater intrusion induced by tides illustrate that the groundwater levels and concentrations are affected significantly near the Dasha river estuarine, which indicates that a very close hydraulic connection exists between the Dasha river and groundwater even considering that anti-seepage measures have been taken beneath the river bed. The numerical results demonstrate also that a decreased tendency of seawater intrusion is expected to occur if groundwater exploitation is not enlarged. A maximum extraction of $1.32 \times$ $10^{4} \mathrm{~m}^{3} / \mathrm{d}$ is obtained by comparing the simulated results from different pumping schemes in the study area.

This study provides a useful example for studying seawater intrusion in coastal aquifers. The model reveals that FEFLOW is an effective and robust simulation tool for studying variable-density flow and transport processes. However, large-scale numerical dispersion is not addressed in this study, and it is necessary to perform a long-term seawater intrusion in the study area.

\section{Acknowledgements}

This work was supported by contract number 08-133, on the numerical simulation of geological disasters induced by seawater intrusion in Shenzhen city. Special thanks are given to the anonymous reviewers for their valuable comments and suggestions which improved the paper.

\section{References}

Aharmouch A and Larabi A 2004 A 3D finite element model for seawater intrusion in coastal aquifers; Develop. Water Sci. 55 1655-1667.

Andersen P F, Mercer J W and White H O 1988 Numerical modelling of salt-water intrusion at Hallandale, Florida; Ground Water 26(5) 619-630.

Bear J 1979 Hydraulics of Groundwater; New York: Mc Graw-Hill.

Chen K P and Jiao J J 2007 Preliminary study on seawater intrusion and aquifer freshening near reclaimed coastal area of Shenzhen, China, Water Science and Technology; Water Supply 2(7) 137-145.

Diersch H J G 2002 'FEFLOW reference manual', finite element subsurface flow \& transport simulation system; WASY Institute for Water Resource Planning and Systems Research Ltd., Berlin, 278p.

Diersch H J G and Kolditz O 2002 Variable-density flow and transport in porous media: Approaches and challenges; Adv. Water Resour. 25 899-944.

Giambastiani B M S, Antonellini M, Oude Essink G H P and Stuurman R J 2007 Saltwater intrusion in the unconfined coastal aquifer of Ravenna (Italy): A numerical model; J. Hydrol. 340 91-104.

Hany F A and Akbar A J 2011 A cost-effective method to control seawater intrusion in coastal aquifers; Water Resour. Manage., doi: 10.1007/s11269-011-9837-7.

Henry H R 1964 Effects of dispersion on salt encroachment in coastal aquifers; In: Sea in coastal aquifers (ed.) Cooper H H et al., US Geological Survey Water Supply Paper 1613-C 70-84.

Huyakorn P S, Anderson P E and Mercer J V P et al. 1987 Saltwater intrusion in aquifers: Development and testing of a three-dimensional finite element model; Water Resour. Res. 23(2) 293-312.

Hu L T and Jiao J J 2010 Modelling the influences of land reclamation on groundwater systems: A case study in Shekou peninsula, Shenzhen, China; Eng. Geol. 114(3-4) 144-153.

Jin L, Blake S, Zheng C and Wu J 2009 A modelling study of seawater intrusion in Alabama Gulf Coast, USA; Environ. Geol. 57 119-130.

Langevin C D 2003 Simulation of submarine groundwater discharge to a marine estuary: Biscayne Bay, Florida; Ground Water 41(6) 758-771.

Langevin C D, Swain E and Wolfert M 2005 Simulation of integrated surface-water/ground-water flow and salinity for a coastal wetland and adjacent estuary; J. Hydrol. 314 212-234.

Mao X, Prommer H, Barrya D A, Langevin C D, Panteleit B and Li L 2006 Three-dimensional model for multi-component reactive transport with variable density groundwater flow; Environ. Model. Softw. 21 615-628.

Oude Essink G H P 2001 Salt water intrusion in a threedimensional groundwater system in The Netherlands: A numerical study; Transp. Porous Media 43 137-158.

Paniconi C, Khlaifi I, Lecca G, Giacomelli A and Tarhouni J 2001 A modelling study of seawater intrusion in the Korba Coastal Plain, Tunisia; Phys. Chem. Earth 26(4) 345-351.

Perera E D P, Jinno K, Tsutsumi A and Hiroshiro Y 2008 Development and verification of a three dimensional density dependent solute transport model for seawater intrusion; Memoirs of the Faculty of Engineering, Kyushu University 68(2) 93-106.

Perera E D P, Jinno K, Tsutsumi A and Hiroshiro Y 2009 Numerical study of salinity variation in a coastal aquifer: A case study of the Motooka region in western Japan; Stoch. Env. Res. Risk A 23 957-965.

Perera E D P, Jinno K, Tsutsumi A and Hiroshiro Y 2010 A numerical study of the saline contamination of a coastal aquifer; Proceedings of the ICE - Water Management 163(7) 367-375.

Pinder G F and Cooper H H 1970 A numerical technique for calculating the transition position of the seawater front; Water Resour. Res. 6-3 875-882.

Qahman K and Larabi A 2006 Evaluation and numerical modelling of seawater intrusion in the Gaza aquifer (Palestine); Hydrogeol. J. 14 713- 728.

Sun N 1981 Numerical model and mathematical method of groundwater flow (Beijing, China: Geological Press).

Shoemaker W B and Edwards K M 2003 Potential for saltwater intrusion into the lower Tamiami Aquifer near Bonita Springs, South Western Florida; U.S. Geological Survey Water-Resources Investigations Report 03-4262.

Sanford W E and Pope J P 2010 Current challenges using models to forecast seawater intrusion: Lessons from the Eastern Shore of Virginia, USA; Hydrogeol. J. 18 $73-93$.

Van Meir N V and Lebbe L 2002 3D density-dependent modelling of sea-level rise scenarios around De Haan 
(Belgium); 17th Salt Water Intrusion Meeting, Delft, The Netherlands, pp. 73-81.

Werner A D and Gallagher M R 2006 Characterization of sea-water intrusion in the Pioneer Valley, Australia using hydrochemistry and three-dimensional numerical modelling; Hydrogeol. J. 14 1452-1469.

Werner A D and Simmons C T 2008 Impact of sea-level rise on sea water intrusion in coastal aquifers; Ground Water 47(2) 197-204.
Xue Y, Xie C and Wu J 1999 Seawater intrusion and research on movement of interface of fresh-saline water (Nanjing, China: Nanjing University Press).

Yuan Y and Liang D 2001 Afterwards prediction of seawater intrusion and controlling engineering; Appl. Math. Mech. 22(11) 163-171.

Zhang Q, Volker R E and Lockington D A 2004 Numerical investigation of seawater intrusion at Gooburrum, Bundaberg, Queensland, Australia; Hydrogeol. J. 12 674-687.

MS received 14 March 2012; revised 16 September 2012; accepted 23 September 2012 